\title{
A New Family of Iterative Methods Based on an Exponential Model for Solving Nonlinear Equations
}

\author{
Tianbao Liu, ${ }^{1}$ Hengyan $\mathrm{Li}^{2}{ }^{2}$ and Zaixiang Pang ${ }^{3}$ \\ ${ }^{1}$ School of Basic Science, Changchun University of Technology, Changchun 130012, China \\ ${ }^{2}$ College of Mathematics and Information Science, North China University of Water Resources and Electric Power, \\ Zhengzhou 450011, China \\ ${ }^{3}$ Engineering Training Center, Changchun University of Technology, Changchun 130012, China
}

Correspondence should be addressed to Tianbao Liu; liujiapeng27@126.com

Received 17 September 2013; Accepted 2 November 2013

Academic Editor: Yongkun Li

Copyright (C) 2013 Tianbao Liu et al. This is an open access article distributed under the Creative Commons Attribution License, which permits unrestricted use, distribution, and reproduction in any medium, provided the original work is properly cited.

We present two new families of iterative methods for obtaining simple roots of nonlinear equations. The first family is developed by fitting the model $m(x)=e^{p x}\left(A x^{2}+B x+C\right)$ to the function $f(x)$ and its derivative $f^{\prime}(x), f^{\prime \prime}(x)$ at a point $x_{n}$. In order to remove the second derivative of the first methods, we construct the second family of iterative methods by approximating the equation $f(x)=0$ around the point $\left(x_{n}, f\left(x_{n}\right)\right)$ by the quadratic equation. Analysis of convergence shows that the new methods have thirdorder or higher convergence. Numerical experiments show that new iterative methods are effective and comparable to those of the well-known existing methods.

\section{Introduction}

In this paper, we consider iterative methods to find a simple root $\alpha$, that is, $f(\alpha)=0$ and $f^{\prime}(\alpha) \neq 0$, of a nonlinear equation:

$$
f(x)=0,
$$

where $f: I \subset R \rightarrow R$ for an open interval $I$ is a scalar function.

Many of the complex problems in science and engineering contains the function of nonlinear and transcendental nature in (1), so finding the simple roots of the nonlinear equation is one of the most important problems in numerical analysis. Numerical iterative methods are often used to obtain the approximate solution of such problems because it is not always possible to obtain its exact solution by usual algebraic process. We all know that Newton's method is an important and basic approach for solving nonlinear equations $[1,2]$, its formulation is given by

$$
x_{n+1}=x_{n}-\frac{f\left(x_{n}\right)}{f^{\prime}\left(x_{n}\right)},
$$

and this method converges quadratically. Earlier, many investigations [3-21] have been made to explain the root of nonlinear algebraic and transcendental equations.

The outline of the paper is as follows. In Section 2, we firstly describe a one-parameter family of third-order methods by fitting the model $m(x)=e^{p x}\left(A x^{2}+B x+C\right)$ to the function $f(x)$ and its derivative $f^{\prime}(x), f^{\prime \prime}(x)$ at a point $x_{n}$, and then we use a quadratic equation for approximating the equation $f(x)=0$ to obtain a four-parameter family of second-derivative-free iterative methods. In Section 3, we obtain some different iterative methods by taking several parameters. In Section 4 , different numerical tests confirm the theoretical results, and the new methods are comparable with other known methods and give better results in many cases. Finally, we infer some conclusions.

\section{Development of Methods and Convergence Analysis}

Consider the exponential model:

$$
m(x)=e^{p x}\left(A x^{2}+B x+C\right),
$$


where $A, B, C$, and $p$ are parameters. We construct a new iteration scheme by fitting model (3) to the function $f(x)$ and its derivative $f^{\prime}(x)$ and $f^{\prime \prime}(x)$ at a point $x_{n}$. Imposing the conditions as follows

$$
\begin{aligned}
& m\left(x_{n}\right)=f\left(x_{n}\right), \\
& m^{\prime}\left(x_{n}\right)=f^{\prime}\left(x_{n}\right), \\
& m^{\prime \prime}\left(x_{n}\right)=f^{\prime \prime}\left(x_{n}\right)
\end{aligned}
$$

at the point $\left(x_{n}, m\left(x_{n}\right)\right)$ and then solving (3) and (4) for $A, B$, and $C$, we have

$$
\begin{gathered}
A=\frac{1}{2}\left[f^{\prime \prime}\left(x_{n}\right)+p^{2} f\left(x_{n}\right)-2 p f^{\prime}\left(x_{n}\right)\right] e^{-p x_{n}}, \\
B=\left[f^{\prime}\left(x_{n}\right)-p f\left(x_{n}\right)\right. \\
\left.\quad-\left(f^{\prime \prime}\left(x_{n}\right)+p^{2} f\left(x_{n}\right)-2 p f^{\prime}\left(x_{n}\right)\right) x_{n}\right] e^{-p x_{n}}, \\
C=\left[f\left(x_{n}\right)-\frac{1}{2}\left[f^{\prime \prime}\left(x_{n}\right)+p^{2} f\left(x_{n}\right)-2 p f^{\prime}\left(x_{n}\right)\right] x_{n}^{2}\right. \\
-\left[f^{\prime}\left(x_{n}\right)-p f\left(x_{n}\right)\right. \\
\left.\left.\quad-\left(f^{\prime \prime}\left(x_{n}\right)+p^{2} f\left(x_{n}\right)-2 p f^{\prime}\left(x_{n}\right)\right) x_{n}\right] x_{n}\right] e^{-p x_{n}} .
\end{gathered}
$$

From (3), (4), and (5), we take the values of $A, B$, and $C$ into (3) and give

$$
\begin{aligned}
m(x) & -f\left(x_{n}\right) e^{p x-p x_{n}} \\
= & \frac{1}{2}\left[f^{\prime \prime}\left(x_{n}\right)+p^{2} f\left(x_{n}\right)-2 p f^{\prime}\left(x_{n}\right)\right]\left(x-x_{n}\right)^{2} e^{p x-p x_{n}} \\
& +\left[f^{\prime}\left(x_{n}\right)-p f\left(x_{n}\right)\right]\left(x-x_{n}\right) e^{p x-p x_{n}}
\end{aligned}
$$

At the root estimate $x_{n+1}$, it follows that $m\left(x_{n+1}\right)=0$. We consider reducing (8) as follows

$$
\begin{aligned}
& \frac{1}{2}\left[f^{\prime \prime}\left(x_{n}\right)+p^{2} f\left(x_{n}\right)-2 p f^{\prime}\left(x_{n}\right)\right]\left(x-x_{n}\right)^{2} \\
& \quad+\left[f^{\prime}\left(x_{n}\right)-p f\left(x_{n}\right)\right]\left(x-x_{n}\right)+f\left(x_{n}\right)=0 .
\end{aligned}
$$

Thus from (9) we develop the iteration formula:

$$
=x_{n}-\frac{\left[f^{\prime}\left(x_{n}\right)-p f\left(x_{n}\right)\right]-f^{\prime}\left(x_{n}\right) \sqrt{1-L_{f, p}\left(x_{n}\right)}}{f^{\prime \prime}\left(x_{n}\right)+p^{2} f\left(x_{n}\right)-2 p f^{\prime}\left(x_{n}\right)},
$$

where

$$
\begin{aligned}
& L_{f, p}\left(x_{n}\right) \\
& \qquad=\frac{2 f^{\prime \prime}\left(x_{n}\right) f\left(x_{n}\right)+p^{2} f^{2}\left(x_{n}\right)-2 p f^{\prime}\left(x_{n}\right) f\left(x_{n}\right)}{f^{\prime 2}\left(x_{n}\right)} .
\end{aligned}
$$

The square root is required in (10); however, this may cost expensively and even fail in the case $1-L_{f, p}\left(x_{n}\right)<0$. In order to avoid the calculation of the square roots, we will derive some forms free from square roots by Taylor approximation $[5]$.

It is easy to know that Taylor approximation of $\sqrt{1-L_{f, p}\left(x_{n}\right)}$ is

$$
\sqrt{1-L_{f, p}\left(x_{n}\right)}=\sum_{k \geq 0}\left(\begin{array}{c}
\frac{1}{2} \\
k
\end{array}\right)\left(-L_{f, p}\left(x_{n}\right)\right)^{k} .
$$

Using (12) in (10), we can obtain the following form:

$$
\begin{aligned}
x_{n+1}=x_{n}- & \left(\left[f^{\prime}\left(x_{n}\right)-p f\left(x_{n}\right)\right]\right. \\
& \left.-f^{\prime}\left(x_{n}\right) \sum_{k \geq 0}^{m}\left(\begin{array}{l}
\frac{1}{2} \\
k
\end{array}\right)\left(-L_{f, p}\left(x_{n}\right)\right)^{k}\right) \\
& \left.\times\left(f^{\prime \prime}\left(x_{n}\right)+p^{2} f\left(x_{n}\right)-2 p f^{\prime}\left(x_{n}\right)\right)^{-1}\right) .
\end{aligned}
$$

We have the convergence analysis of the methods by (13).

Theorem 1. Let $\alpha \in I$ be a simple zero of sufficiently differentiable function $f: I \subset R \rightarrow R$ for an open interval I. If $x_{0}$ is sufficiently close to $\alpha$, for $m \geq 1$, the methods defined by (13) are cubically convergent.

Proof. Let $e_{n}=x_{n}-\alpha$, and we use the following Taylor expansions:

$$
f\left(x_{n}\right)=f^{\prime}(\alpha)\left[e_{n}+c_{2} e_{n}^{2}+c_{3} e_{n}^{3}+c_{4} e_{n}^{4}+O\left(e_{n}^{5}\right)\right],
$$

where $c_{k}=(1 / k !)\left(f^{(k)}(\alpha) / f^{\prime}(\alpha)\right)$; furthermore, we have

$$
\begin{aligned}
f^{\prime}\left(x_{n}\right)=f^{\prime}(\alpha)[ & 1+2 c_{2} e_{n}+3 c_{3} e_{n}^{2}+4 c_{4} e_{n}^{3} \\
& \left.+5 c_{5} e_{n}^{4}+O\left(e_{n}^{5}\right)\right], \\
f^{\prime \prime}\left(x_{n}\right)=f^{\prime}(\alpha)[ & 2 c_{2}+6 c_{3} e_{n}+12 c_{4} e_{n}^{2}+20 c_{5} e_{n}^{3} \\
& \left.+30 c_{6} e_{n}^{4}+O\left(e_{n}^{5}\right)\right] .
\end{aligned}
$$

Since (15), we obtain

$$
\begin{aligned}
f^{\prime 2}\left(x_{n}\right)=f^{\prime 2}(\alpha)[ & 1+4 c_{2} e_{n}+\left(6 c_{3}+4 c_{2}^{2}\right) e_{n}^{2} \\
& +\left(8 c_{4}+12 c_{2} c_{3}\right) e_{n}^{3} \\
& \left.+\left(10 c_{5}+16 c_{2} c_{4}+9 c_{3}^{2}\right) e_{n}^{4}+O\left(e_{n}^{5}\right)\right] .
\end{aligned}
$$

Since (14), we get

$$
f^{2}\left(x_{n}\right)=f^{\prime 2}(\alpha)\left[e_{n}^{2}+2 c_{2} e_{n}^{3}+\left(2 c_{3}+c_{2}^{2}\right) e_{n}^{4}+O\left(e_{n}^{5}\right)\right] .
$$


From (14) and (15), we also easily have

$$
\begin{aligned}
f\left(x_{n}\right) f^{\prime}\left(x_{n}\right)=f^{\prime 2}(\alpha) & {\left[e_{n}+3 c_{2} e_{n}^{2}+\left(2 c_{2}^{2}+4 c_{3}\right) e_{n}^{3}\right.} \\
& \left.+\left(5 c_{4}+5 c_{2} c_{3}\right) e_{n}^{4}+O\left(e_{n}^{5}\right)\right] .
\end{aligned}
$$

We obtain the following expression by taking into account (14) and (16):

$$
\begin{aligned}
& f\left(x_{n}\right) f^{\prime \prime}\left(x_{n}\right) \\
&=f^{\prime 2}(\alpha) {\left[2 c_{2} e_{n}+\left(2 c_{2}^{2}+6 c_{3}\right) e_{n}^{2}+\left(8 c_{2} c_{3}+12 c_{4}\right) e_{n}^{3}\right.} \\
&\left.+\left(14 c_{2} c_{4}+6 c_{3}^{2}+20 c_{5}\right) e_{n}^{4}+O\left(e_{n}^{5}\right)\right] .
\end{aligned}
$$

From (18), (19), and (20), we obtain

$$
\begin{aligned}
2 f^{\prime \prime}\left(x_{n}\right) f\left(x_{n}\right)+p^{2} f^{2}\left(x_{n}\right)-2 p f^{\prime}\left(x_{n}\right) f\left(x_{n}\right) & \\
=f^{\prime 2}(\alpha)[ & \left(4 c_{2}-2 p\right) e_{n}+\left(4 c_{2}^{2}+12 c_{3}+p^{2}-6 p c_{2}\right) e_{n}^{2} \\
+ & \left(16 c_{2} c_{3}+24 c_{4}+2 p^{2} c_{2}-4 p c_{2}^{2}-8 p c_{3}\right) e_{n}^{3} \\
+ & \left(28 c_{2} c_{4}+12 c_{3}^{2}+40 c_{5}+2 p^{2} c_{3}+p^{2} c_{2}^{2}\right. \\
& \left.\left.-10 p c_{4}-10 p c_{2} c_{3}\right) e_{n}^{4}+O\left(e_{n}^{5}\right)\right] .
\end{aligned}
$$

From (17) and (21), we have

$$
\begin{aligned}
L_{f, p}\left(x_{n}\right)=f^{\prime}(\alpha)[ & \left(4 c_{2}-2 p\right) e_{n} \\
+ & \left(12 c_{3}-12 c_{2}^{2}+p^{2}+2 p c_{2}\right) e_{n}^{2} \\
+ & \left(24 c_{4}-56 c_{2} c_{3}-2 p^{2} c_{2}-4 p c_{2}^{2}\right. \\
& \left.\left.+4 p c_{3}+32 c_{2}^{3}\right) e_{n}^{3}+O\left(e_{n}^{4}\right)\right] .
\end{aligned}
$$

From (14) and (15), we have

$$
\begin{aligned}
& f^{\prime}\left(x_{n}\right)-p f\left(x_{n}\right) \\
& =f^{\prime}(\alpha)\left[1+\left(2 c_{2}-p\right) e_{n}+\left(3 c_{3}-p c_{2}\right) e_{n}^{2}\right. \\
& \left.+\left(4 c_{4}-p c_{3}\right) e_{n}^{3}+\left(5 c_{5}-p c_{4}\right) e_{n}^{4}+O\left(e_{n}^{5}\right)\right] .
\end{aligned}
$$

Using (14), (15), and (16), we have

$$
\begin{array}{rl}
f^{\prime \prime}\left(x_{n}\right)+p^{2} & f\left(x_{n}\right)-2 p f^{\prime}\left(x_{n}\right) \\
=f^{\prime}(\alpha)[ & \left(2 c_{2}-2 p\right)+\left(6 c_{3}+p^{2}-4 p c_{2}\right) e_{n} \\
& +\left(12 c_{4}+p^{2} c_{2}-6 p c_{3}\right) e_{n}^{2}+\left(20 c_{5}+p^{2} c_{3}-8 p c_{4}\right) e_{n}^{3} \\
& \left.+\left(30 c_{6}+p^{2} c_{4}-10 p c_{5}\right) e_{n}^{4}+O\left(e_{n}^{5}\right)\right] .
\end{array}
$$

We know that

$$
\begin{aligned}
\sum_{k \geq 0}^{m}\left(\begin{array}{c}
\frac{1}{2} \\
k
\end{array}\right)\left(-L_{f, p}\left(x_{n}\right)\right)^{k} \\
=1-\frac{1}{2} L_{f, p}\left(x_{n}\right)-\frac{1}{8} L_{f, p}\left(x_{n}\right)^{2}-\frac{1}{16} L_{f, p}\left(x_{n}\right)^{3}+\cdots \\
=1-\left(2 c_{2}-p\right) e_{n}+\left(p c_{2}+4 c_{2}^{2}-6 c_{3}-p^{2}\right) e_{n}^{2} \\
\quad-\left(2 p^{2} c_{2}-16 c_{2} c_{3}-4 p c_{3}+12 c_{4}+8 c_{2}^{3}-p^{3}\right) e_{n}^{3} \\
+O\left(e_{n}^{4}\right) .
\end{aligned}
$$

From (15) and (25), we obtain

$$
\begin{aligned}
f^{\prime}\left(x_{n}\right) \sum_{k \geq 0}^{m}\left(\begin{array}{c}
\frac{1}{2} \\
k
\end{array}\right)\left(-L_{f, p}\left(x_{n}\right)\right)^{k} \\
=f^{\prime}(\alpha)\left[1+p e_{n}+\left(3 p c_{2}-p^{2}-3 c_{3}\right) e_{n}^{2}\right. \\
+\left(7 p c_{3}-4 p^{2} c_{2}-2 c_{2} c_{3}+p^{3}-8 c_{4}+2 p c_{2}^{2}\right) e_{n}^{3} \\
\left.+O\left(e_{n}^{4}\right)\right] .
\end{aligned}
$$

From (23) and (26), we have

$$
\begin{aligned}
& {\left[f^{\prime}\left(x_{n}\right)-p f\left(x_{n}\right)\right]-f^{\prime}\left(x_{n}\right) \sum_{k \geq 0}^{m}\left(\begin{array}{c}
\frac{1}{2} \\
k
\end{array}\right)\left(-L_{f, p}\left(x_{n}\right)\right)^{k}} \\
& =2\left(c_{2}-p\right) e_{n}-\left(4 p c_{2}-6 c_{3}-p^{2}\right) e_{n}^{2} \\
& \quad-\left(8 p c_{3}-12 c_{4}-4 p^{2} c_{2}-2 c_{2} c_{3}+p^{3}+2 p c_{2}^{2}\right) e_{n}^{3} \\
& \quad+O\left(e_{n}^{4}\right) .
\end{aligned}
$$

Using (13), (24), and (27), we have

$$
\begin{gathered}
x_{n+1}=x_{n}-\left(\left(\left[f^{\prime}\left(x_{n}\right)-p f\left(x_{n}\right)\right]\right.\right. \\
\left.-f^{\prime}\left(x_{n}\right) \sum_{k \geq 0}^{m}\left(\begin{array}{c}
\frac{1}{2} \\
k
\end{array}\right)\left(-L_{f, p}\left(x_{n}\right)\right)^{k}\right) \\
\left.\times\left(f^{\prime \prime}\left(x_{n}\right)+p^{2} f\left(x_{n}\right)-2 p f^{\prime}\left(x_{n}\right)\right)^{-1}\right) \\
=x_{n}-e_{n}-\left(\frac{1}{2} p^{2}+c_{3}-p c_{2}\right) e_{n}^{3}+O\left(e_{n}^{4}\right) .
\end{gathered}
$$

From $e_{n+1}=x_{n+1}-\alpha$, we have

$$
e_{n+1}=\left(p c_{2}-\frac{1}{2} p^{2}-c_{3}\right) e_{n}^{3}+O\left(e_{n}^{4}\right),
$$

which completes the proof. 
The family methods given by (13) are novel third-order methods, but the methods depend on the second derivatives in computing process, and therefore their practical applications are restricted in some cases. In recent years, several methods with free second derivatives have been developed; see [4-15] and references therein.

In order to avoid the calculation of the second derivatives, we consider approximating the equation $f(x)=0$ around the point $\left(x_{n}, f\left(x_{n}\right)\right)$ by the quadratic equation in $x$ and $y$ in the following form [8]:

$$
a_{1} x^{2}+a_{2} y^{2}+a_{3} x y+a_{4} x+a_{5} y+a_{6}=0
$$

where $a_{i} \in R, i=1,2, \ldots, 6$, are parameters. We impose the tangency conditions

$$
\begin{aligned}
& y\left(x_{n}\right)=f\left(x_{n}\right), \\
& y^{\prime}\left(x_{n}\right)=f^{\prime}\left(x_{n}\right), \\
& y\left(w_{n}\right)=f\left(w_{n}\right),
\end{aligned}
$$

where $x_{n}$ is $n$th iterate and

$$
w_{n}=x_{n}-\frac{f\left(x_{n}\right)}{f^{\prime}\left(x_{n}\right)} .
$$

From (30) and (31), we have

$$
\begin{aligned}
& f^{\prime \prime}\left(x_{n}\right) \\
& \approx y^{\prime \prime}\left(x_{n}\right) \\
& =L_{a_{i}}\left(x_{n}, w_{n}\right) \\
& =\left(\left[2 a_{1}+2 a_{2} f^{\prime 2}\left(x_{n}\right)+2 a_{3} f^{\prime}\left(x_{n}\right)\right] f^{\prime 2}\left(x_{n}\right) f\left(w_{n}\right)\right) \\
& \quad \times\left(a_{1} f^{2}\left(x_{n}\right)+a_{2} f^{\prime 2}\left(x_{n}\right)\left[f\left(x_{n}\right)-f\left(w_{n}\right)\right]^{2}\right. \\
& \left.\quad+a_{3} f\left(x_{n}\right) f^{\prime}\left(x_{n}\right)\left[f\left(x_{n}\right)-f\left(w_{n}\right)\right]\right)^{-1},
\end{aligned}
$$

where $i=1,2,3$.

From (33) we can approximate

$$
\begin{aligned}
& L_{a_{i}, f, p}\left(x_{n}, w_{n}\right) \\
& =\frac{2 L_{a_{i}}\left(x_{n}, w_{n}\right) f\left(x_{n}\right)+p^{2} f^{2}\left(x_{n}\right)-2 p f^{\prime}\left(x_{n}\right) f\left(x_{n}\right)}{f^{\prime 2}\left(x_{n}\right)} .
\end{aligned}
$$

Using $L_{a_{i}, f, p}\left(x_{n}, w_{n}\right)$ instead of $L_{f, p}\left(x_{n}\right)(11)$, we obtain a new four-parameter family of methods free from second derivative:

$$
\begin{aligned}
x_{n+1}=x_{n}-( & \left(\left[f^{\prime}\left(x_{n}\right)-p f\left(x_{n}\right)\right]\right. \\
& \left.-f^{\prime}\left(x_{n}\right) \sum_{k \geq 0}^{m}\left(\begin{array}{c}
\frac{1}{2} \\
k
\end{array}\right)\left(-L_{a_{i} f, p}\left(x_{n}, w_{n}\right)\right)^{k}\right) \\
& \left.\times\left(L_{a_{i}}\left(x_{n}, w_{n}\right)+p^{2} f\left(x_{n}\right)-2 p f^{\prime}\left(x_{n}\right)\right)^{-1}\right),
\end{aligned}
$$

where $a_{i}(i=1,2,3), p \in R, m \geq 0$.

We also have the convergence analysis of the methods by (35).

Theorem 2. Let $\alpha \in I$ be a simple zero of sufficiently differentiable function $f: I \subset R \rightarrow R$ for an open interval $I$. If $x_{0}$ is sufficiently close to $\alpha$, for $m \geq 1, a_{i}(i=1,2,3), p \in R$, the methods defined by (35) are at least cubically convergent; as particular cases, if $m \geq 2, a_{2}=a_{3}=p=0, a_{1} \in R$ and the methods have convergence order four.

Proof. Let $e_{n}=x_{n}-\alpha$, and we use the following Taylor expansions:

$$
f\left(x_{n}\right)=f^{\prime}(\alpha)\left[e_{n}+c_{2} e_{n}^{2}+c_{3} e_{n}^{3}+c_{4} e_{n}^{4}+O\left(e_{n}^{5}\right)\right],
$$

where $c_{k}=(1 / k !)\left(f^{(k)}(\alpha) / f^{\prime}(\alpha)\right)$; furthermore, we have

$$
\begin{aligned}
& f^{2}\left(x_{n}\right) \\
& \quad=f^{\prime 2}(\alpha)\left[e_{n}^{2}+2 c_{2} e_{n}^{3}+\left(2 c_{3}+c_{2}^{2}\right) e_{n}^{4}+O\left(e_{n}^{5}\right)\right], \\
& f^{\prime}\left(x_{n}\right) \\
& \quad=f^{\prime}(\alpha)\left[1+2 c_{2} e_{n}+3 c_{3} e_{n}^{2}+4 c_{4} e_{n}^{3}+5 c_{5} e_{n}^{4}+O\left(e_{n}^{5}\right)\right] .
\end{aligned}
$$

Dividing (36) by (38)

$$
\begin{aligned}
\frac{f\left(x_{n}\right)}{f^{\prime}\left(x_{n}\right)}= & e_{n}-c_{2} e_{n}^{2}+2\left(c_{2}^{2}-c_{3}\right) e_{n}^{3} \\
& +\left(7 c_{2} c_{3}-4 c_{2}^{3}-3 c_{4}\right) e_{n}^{4}+O\left(e_{n}^{5}\right) .
\end{aligned}
$$

From (39), we get

$$
\begin{aligned}
w_{n}= & x_{n}-\frac{f\left(x_{n}\right)}{f^{\prime}\left(x_{n}\right)} \\
= & \alpha+c_{2} e_{n}^{2}-2\left(c_{2}^{2}-c_{3}\right) e_{n}^{3} \\
& -\left(7 c_{2} c_{3}-4 c_{2}^{3}-3 c_{4}\right) e_{n}^{4}+O\left(e_{n}^{5}\right) .
\end{aligned}
$$


Expanding $f\left(w_{n}\right)$ in Taylor's Series about $\alpha$ and using (40), we get

$$
\begin{aligned}
f\left(w_{n}\right)=f^{\prime}(\alpha)[ & w_{n}-\alpha+c_{2}\left(w_{n}-\alpha\right)^{2} \\
& \left.+c_{3}\left(w_{n}-\alpha\right)^{3}+c_{4}\left(w_{n}-\alpha\right)^{4}+\cdots\right] \\
=f^{\prime}(\alpha)[ & c_{2} e_{n}^{2}+2\left(c_{3}-c_{2}^{2}\right) e_{n}^{3} \\
& \left.+\left(5 c_{2}^{3}+3 c_{4}-7 c_{2} c_{3}\right) e_{n}^{4}+O\left(e_{n}^{5}\right)\right] .
\end{aligned}
$$

From (36) and (41), we have

$$
f\left(x_{n}\right) f\left(w_{n}\right)=f^{\prime 2}(\alpha)\left[c_{2} e_{n}^{3}+\left(2 c_{3}-c_{2}^{2}\right) e_{n}^{4}+O\left(e_{n}^{5}\right)\right] .
$$

From (38), we obtain

$$
\begin{aligned}
f^{\prime 2}\left(x_{n}\right)=f^{\prime 2}(\alpha)[ & 1+4 c_{2} e_{n}+\left(6 c_{3}+4 c_{2}^{2}\right) e_{n}^{2} \\
& +\left(8 c_{4}+12 c_{2} c_{3}\right) e_{n}^{3} \\
& \left.+\left(10 c_{5}+16 c_{2} c_{4}+9 c_{3}^{2}\right) e_{n}^{4}+O\left(e_{n}^{5}\right)\right] .
\end{aligned}
$$

From (38), (41), and (43) we also easily obtain

$$
\begin{gathered}
{\left[2 a_{1}+2 a_{2} f^{\prime 2}\left(x_{n}\right)+2 a_{3} f^{\prime}\left(x_{n}\right)\right] f^{\prime 2}\left(x_{n}\right) f\left(w_{n}\right)} \\
=f^{\prime 3}(\alpha)\left[2\left(a_{2} f^{\prime 2}(\alpha)+a_{3} f^{\prime}(\alpha)+a_{1}\right) c_{2} e_{n}^{2}\right. \\
+4\left(a_{2} f^{\prime 2}(\alpha) c_{3}+3 a_{2} f^{\prime 2}(\alpha) c_{2}^{2}+a_{3} f^{\prime}(\alpha) c_{3}\right. \\
\left.+2 f^{\prime}(\alpha) a_{3} c_{2}^{2}+a_{1} c_{3}+a_{1} c_{2}^{2}\right) e_{n}^{3} \\
+2\left(3 a_{2} f^{\prime 2}(\alpha) c_{4}+21 f^{\prime 2}(\alpha) a_{2} c_{2} c_{3}\right. \\
+5 f^{\prime}(\alpha) a_{3} c_{2}^{3}+3 a_{3} f^{\prime}(\alpha) c_{4} \\
\left.\left.+14 f^{\prime}(\alpha) a_{3} c_{2} c_{3}+7 a_{1} c_{3} c_{2}\right) e_{n}^{4}+O\left(e_{n}^{5}\right)\right]
\end{gathered}
$$

Substituting (36), (37), (38), (41), and (43) in the denominator of $L_{a_{i}}\left(x_{n}, w_{n}\right)$, we obtain

$$
\begin{aligned}
a_{1} f^{2}\left(x_{n}\right)+a_{2} f^{\prime 2}\left(x_{n}\right)\left[f\left(x_{n}\right)-f\left(w_{n}\right)\right]^{2} \\
+a_{3} f\left(x_{n}\right) f^{\prime}\left(x_{n}\right)\left[f\left(x_{n}\right)-f\left(w_{n}\right)\right] \\
=f^{\prime 2}(\alpha)\left[\left(a_{1}+a_{2} f^{\prime 2}(\alpha)+a_{3} f^{\prime}(\alpha)\right) e_{n}^{2}\right. \\
+\left(2 a_{1} c_{2}+4 a_{2} c_{2} f^{\prime 2}(\alpha)+3 a_{3} c_{2} f^{\prime}(\alpha)\right) e_{n}^{3} \\
+\left(2 a_{1} c_{3}+a_{1} c_{2}^{2}+8 a_{2} c_{2}^{2} f^{\prime 2}(\alpha)+4 a_{2} c_{3} f^{\prime 2}(\alpha)\right. \\
\left.\left.+4 a_{3} c_{2}^{2} f^{\prime}(\alpha)+3 a_{3} c_{3} f^{\prime}(\alpha)\right) e_{n}^{4}+O\left(e_{n}^{5}\right)\right] .
\end{aligned}
$$

Using (44) and (45), we have

$$
\begin{gathered}
L_{a_{i}}\left(x_{n}, w_{n}\right) \\
=f^{\prime}(\alpha)\left[2 c_{2}+2\left(2 a_{2} f^{\prime 2}(\alpha) c_{3}+2 a_{2} f^{\prime 2}(\alpha) c_{2}^{2}\right.\right. \\
+2 a_{3} f^{\prime}(\alpha) c_{3}+f^{\prime}(\alpha) a_{3} c_{2}^{2} \\
\left.+2 a_{1} c_{3}\right) e_{n} /\left(a_{2} f^{\prime 2}(\alpha)\right. \\
\left.+a_{3} f^{\prime}(\alpha)+a_{1}\right) \\
-\left(+16 a_{2}^{2} f^{\prime 4}(\alpha) c_{2}^{3}-14 f^{\prime 3}(\alpha) a_{2} c_{2} c_{3} a_{3}\right. \\
-6 a_{2} f^{\prime 3}(\alpha) c_{4} a_{3}-9 f^{\prime 4}(\alpha) a_{2}^{2} c_{2} c_{3} \\
+17 f^{\prime 3}(\alpha) a_{3} c_{2}^{3} a_{2}-5 f^{\prime 2}(\alpha) a_{3}^{2} c_{2} c_{3} \\
-3 a_{2} f^{\prime 2}(\alpha) c_{4} a_{1}+2 f^{\prime}(\alpha) a_{3} c_{2}^{3} a_{1} \\
-3 a_{3} f^{\prime}(\alpha) c_{4} a_{1}+a_{1}^{2} c_{2}^{3}-3 a_{2}^{2} f^{\prime}(\alpha)^{4} c_{4} \\
+2 f^{\prime}(\alpha)^{2} a_{3}^{2} c_{2}^{3}-3 a_{3}^{2} f^{\prime}(\alpha)^{2} c_{4}-a_{1}^{2} c_{3} c_{2} \\
-10 f^{\prime 2}(\alpha) a_{2} c_{2} c_{3} a_{1}-6 f^{\prime}(\alpha) a_{3} c_{2} c_{3} a_{1} \\
\left.+13 a_{1} c_{2}^{3} a_{2} f^{\prime 2}(\alpha)\right) e_{n}^{2} \\
\left.\left.(\alpha)+a_{3} f^{\prime}(\alpha)+a_{1}\right)^{2}+O\left(e_{n}^{3}\right)\right]
\end{gathered}
$$

From (36), (37), and (46), we have

$$
\begin{aligned}
2 L_{a_{i}}\left(x_{n}, w_{n}\right) f\left(x_{n}\right)+p^{2} f^{2}\left(x_{n}\right)-2 p f^{\prime}\left(x_{n}\right) f\left(x_{n}\right) & \\
=f^{\prime 2}(\alpha)\left[2\left(2 c_{2}-p\right) e_{n}\right. & \\
+ & \left(12 a_{2} f^{\prime 2}(\alpha) c_{2}^{2}+8 f^{\prime}(\alpha) a_{3} c_{2}^{2}\right. \\
+ & 4 a_{1} c_{2}^{2}+p^{2} a_{2} f^{\prime}(\alpha)^{2}+p^{2} a_{2} f^{\prime}(\alpha) \\
+ & p^{2} a_{1}+8 a_{2} f^{\prime 2}(\alpha) c_{3}+8 a_{3} f^{\prime}(\alpha) c_{3} \\
+ & 8 a_{1} c_{3}-6 p c_{2} a_{2} f^{\prime 2}(\alpha)-6 p c_{2} a_{3} f^{\prime}(\alpha) \\
& \left.-6 p c_{2} a_{1}\right) e_{n}^{2} /\left(a_{2} f^{\prime 2}(\alpha)+a_{3} f^{\prime}(\alpha)+a_{1}\right) \\
- & \left(2 p c_{2}^{2} c^{2} f^{\prime 2}(\alpha)+2 p c_{2}^{2} a_{2}^{2} f^{\prime 4}(\alpha)\right. \\
& -6 f^{\prime 2}(\alpha) a_{3}^{2} c_{4}-6 f^{\prime 4}(\alpha) a_{2}^{2} c_{4} \\
& +28 f^{\prime 4}(\alpha) a_{2}^{2} c_{2}^{3}+2 a_{1}^{2} c_{2}^{3}-p^{2} c_{2} a_{2}^{2} f^{\prime 4}(\alpha) \\
& -p^{2} c_{2} a_{3}^{2} f^{\prime 2}(\alpha)-2 p^{2} c_{2} a_{2} f^{\prime 3}(\alpha) a_{3} \\
& -2 p^{2} c_{2} a_{2} f^{\prime 2}(\alpha) a_{1}-2 p^{2} c_{2} a_{3} f^{\prime}(\alpha) a_{1}
\end{aligned}
$$




$$
\begin{aligned}
& +2 p c_{2}^{2} a_{1}^{2}+4 p c_{3} a_{1}^{2}+2 f^{\prime}(\alpha)^{2} a_{3}^{2} c_{2}^{3} \\
& -32 f^{\prime 2}(\alpha) a_{2} c_{2} c_{3} a_{1}-16 f^{\prime 2}(\alpha) c^{2} c_{2} c_{3} \\
& -40 f^{\prime 3}(\alpha) a_{2} c_{2} c_{3} a_{3}-6 f^{\prime 2}(\alpha) a_{2} c_{4} a_{1} \\
& +2 f^{\prime}(\alpha) a_{3} c_{2}^{3} a_{1}-4 f^{\prime 2}(\alpha) a_{1} c_{2}^{3} a_{2} \\
& -12 f^{\prime 3}(\alpha) a_{2} c_{4} a_{3}-6 f^{\prime}(\alpha) a_{3} c_{4} a_{1} \\
& -6 f^{\prime}(\alpha)^{3} a_{3} c_{2}^{3} a_{2}-24 f^{\prime}(\alpha) a_{3} c_{2} c_{3} a_{1} \\
& -8 a_{1}^{2} c_{3} c_{2}-24 f^{\prime 4}(\alpha) a_{2}^{2} c_{2} c_{3} \\
& +4 p c_{2}^{2} a_{2} f^{\prime}(\alpha)^{3} a_{3}+4 p c_{2}^{2} a_{2} f^{\prime 2}(\alpha) a_{1} \\
& +4 p c_{2}^{2} a_{3} f^{\prime}(\alpha) a_{1}+8 p c_{3} a_{2} f^{\prime 3}(\alpha) a_{3} \\
& +8 p c_{3} a_{2} f^{\prime 2}(\alpha) a_{1}+8 p c_{3} a_{3} f^{\prime}(\alpha) a_{1} \\
& \left.-p^{2} c_{2} a_{1}^{2}+4 p c_{3} a_{3}^{2} f^{\prime 2}(\alpha)+4 p c_{3} a_{2}^{2} f^{\prime}(\alpha)^{4}\right) e_{n}^{2} \\
& \left./\left(a_{2} f^{\prime 2}(\alpha)+a_{3} f^{\prime}(\alpha)+a_{1}\right)^{2}+O\left(e_{n}^{3}\right)\right] .
\end{aligned}
$$

Since (43) and (47), we get

$$
\begin{aligned}
L_{a_{i}, f, p}\left(x_{n}, w_{n}\right) & \\
=\left[2 \left(2 c_{2}\right.\right. & -p) e_{n} \\
- & \left(4 a_{2} f^{\prime 2}(\alpha) c_{2}^{2}+8 a_{3} f^{\prime}(\alpha) c_{2}^{2}\right. \\
& +12 a_{1} c_{2}^{2}-p^{2} a_{2} f^{\prime 2}(\alpha)-p^{2} a_{3} f^{\prime}(\alpha) \\
& -p^{2} a_{1}-8 a_{2} f^{\prime 2}(\alpha) c_{3}-8 a_{3} f^{\prime}(\alpha) c_{3} \\
& -8 a_{1} c_{3}-2 p c_{2} a_{2} f^{\prime 2}(\alpha)-2 p c_{2} a_{3} f^{\prime}(\alpha) \\
& \left.-2 p c_{2} a_{1}\right) e_{n}^{2} /\left(a_{2} f^{\prime 2}(\alpha)+a_{3} f^{\prime}(\alpha)+a_{1}\right) \\
+2( & -2 p c_{2}^{2} a_{3}^{2} f^{\prime 2}(\alpha)-2 p c_{2}^{2} a_{2}^{2} f^{\prime 4}(\alpha) \\
& +6 f^{\prime 2}(\alpha) a_{3}^{2} c_{4}+6 f^{\prime 4}(\alpha) a_{2}^{2} c_{4} \\
& -28 f^{\prime 4}(\alpha) a_{2}^{2} c_{2}^{3}+14 a_{1}^{2} c_{2}^{3} \\
& -p^{2} c_{2} a_{2}^{2} f^{\prime 4}(\alpha)-p^{2} c_{2} a_{3}^{2} f^{\prime 2}(\alpha) \\
& -2 p^{2} c_{2} a_{2} f^{\prime 3}(\alpha) a_{3}-2 p^{2} c_{2} a_{2} f^{\prime 2}(\alpha) a_{1} \\
& -2 p^{2} c_{2} a_{3} f^{\prime}(\alpha) a_{1}-2 p c_{2}^{2} a_{1}^{2}
\end{aligned}
$$

$$
\begin{aligned}
& +2 p c_{3} a_{1}^{2}+6 f^{\prime 2}(\alpha) a_{3}^{2} c_{2}^{3} \\
& -24 f^{\prime 2}(\alpha) a_{2} c_{2} c_{3} a_{1}-12 f^{\prime 2}(\alpha) a_{3}^{2} c_{2} c_{3} \\
& -16 f^{\prime 3}(\alpha) a_{2} c_{2} c_{3} a_{3}+6 f^{\prime 2}(\alpha) a_{2} c_{4} a_{1} \\
& +22 f^{\prime}(\alpha) a_{3} c_{2}^{3} a_{1}+20 f^{\prime 2}(\alpha) a_{1} c_{2}^{3} a_{2} \\
& +12 f^{\prime 3}(\alpha) a_{2} c_{4} a_{3}+6 f^{\prime}(\alpha) a_{3} c_{4} a_{1} \\
& +14 f^{\prime 3}(\alpha) a_{3} c_{2}^{3} a_{2}-32 f^{\prime}(\alpha) a_{3} c_{2} c_{3} a_{1} \\
& -20 a_{1}^{2} c_{3} c_{2}-4 f^{\prime 4}(\alpha) a_{2}^{2} c_{2} c_{3} \\
& -4 p c_{2}^{2} a_{2} f^{\prime 3}(\alpha) a_{3}-4 p c_{2}^{2} a_{2} f^{\prime 2}(\alpha) a_{1} \\
& -4 p c_{2}^{2} a_{3} f^{\prime}(\alpha) a_{1}+4 p c_{3} a_{2} f^{\prime 3}(\alpha) a_{3} \\
& +4 p c_{3} a_{2} f^{\prime 2}(\alpha) a_{1}+4 p c_{3} a_{3} f^{\prime}(\alpha) a_{1} \\
& -p^{2} c_{2} a_{1}^{2}+2 p c_{3} a_{3}^{2} f^{\prime 2}(\alpha) \\
& \left.+2 p c_{3} a_{2}^{2} f^{\prime 4}(\alpha)\right) e_{n}^{3} \\
& \left./\left(a_{2} f^{\prime 2}(\alpha)+a_{3} f^{\prime}(\alpha)+a_{1}\right)^{2}+O\left(e_{n}^{4}\right)\right] .
\end{aligned}
$$

Using (48), we write

$$
\begin{aligned}
\sum_{k \geq 0}^{m}\left(\begin{array}{c}
\frac{1}{2} \\
k
\end{array}\right)\left(-L_{a_{i}, f, p}\left(x_{n}, w_{n}\right)\right)^{k} & \\
= & 1-\frac{1}{2} L_{a_{i}, f, p}\left(x_{n}, w_{n}\right)-\frac{1}{8} L_{a_{i}, f, p}\left(x_{n}, w_{n}\right)^{2} \\
& -\frac{1}{16} L_{a_{i}, f, p}\left(x_{n}, w_{n}\right)^{3}+\cdots \\
= & 1-\left(2 c_{2}-p\right) e_{n} \\
+ & \frac{1}{2}\left(4 a_{2} f^{\prime 2}(\alpha) c_{2}^{2}+8 c f^{\prime}(\alpha) c_{2}^{2}\right. \\
& +12 a_{1} c_{2}^{2}-p^{2} a_{2} f^{\prime 2}(\alpha)-p^{2} a_{3} f^{\prime}(\alpha) \\
& \quad-p^{2} a_{1}-8 a_{2} f^{\prime 2}(\alpha) c_{3}-8 a_{3} f^{\prime}(\alpha) c_{3} \\
& \quad-8 a_{1} c_{3}-2 p c_{2} a_{2} f^{\prime 2}(\alpha)-2 p c_{2} a_{3} f^{\prime}(\alpha) \\
& \left.\quad-2 p c_{2} a_{1}\right) e_{n}^{2} /\left(a_{2} f^{\prime 2}(\alpha)+a_{3} f^{\prime}(\alpha)+a_{1}\right) \\
+ & \left(e_{n}^{3}\right) .
\end{aligned}
$$


Using (36), (38), and (46), we obtain

$$
\begin{aligned}
& L_{a_{i}}\left(x_{n}, w_{n}\right)+p^{2} f\left(x_{n}\right)-p f^{\prime}\left(x_{n}\right) \\
&=f^{\prime}(\alpha)\left[2\left(c_{2}-p\right)\right. \\
&+\left(4 a_{2} f^{\prime 2}(\alpha) c_{3}\right. \\
&+4 a_{2} f^{\prime 2}(\alpha) c_{2}^{2}+4 a_{3} f^{\prime}(\alpha) c_{3}+2 a_{3} f^{\prime}(\alpha) c_{2}^{2} \\
&+4 a_{1} c_{3}+p^{2} a_{2} f^{\prime 2}(\alpha)+p^{2} a_{3} f^{\prime}(\alpha)+p^{2} a_{1} \\
&\left.-4 p c_{2} a_{2} f^{\prime 2}(\alpha)-4 p c_{2} a_{3} f^{\prime}(\alpha)-4 p c_{2} a_{1}\right) e_{n} \\
& /\left(a_{2} f^{\prime 2}(\alpha)+a_{3} f^{\prime}(\alpha)+a_{1}\right) \\
&-\left(6 p c_{3} a_{1}^{2}-p^{2} c_{2} a_{2}^{2} f^{\prime 4}(\alpha)-p^{2} c_{2} a_{1}^{2}\right. \\
&+6 p f^{\prime 4}(\alpha) c_{3} a_{2}^{2}+6 p f^{\prime 2}(\alpha) c_{3} a_{3}^{2} \\
&-2 p^{2} c_{2} a_{3} f^{\prime}(\alpha) a_{1}-2 p^{2} c_{2} a_{2} f^{\prime 2}(\alpha) a_{1} \\
&-2 p^{2} c_{2} a_{2} f^{\prime 3}(\alpha) a_{3}-p^{2} c_{2} a_{3}^{2} f^{\prime 2}(\alpha) \\
&+12 f^{\prime 3}(\alpha) p c_{3} a_{2} a_{3}+12 p f^{\prime 2}(\alpha) c_{3} a_{2} a_{1} \\
&+12 p f^{\prime}(\alpha) c_{3} a_{3} a_{1}-18 a_{2}^{2} f^{\prime 4}(\alpha) c_{2} c_{3} \\
&+26 a_{1} c_{2}^{3} a_{2} f^{\prime 2}(\alpha)-20 c_{2} a_{2} f^{\prime 2}(\alpha) c_{3} a_{1} \\
&-12 c_{2} a_{3} f^{\prime}(\alpha) c_{3} a_{1}-12 f^{\prime 3}(\alpha) a_{2} c_{4} a_{3} \\
&-6 a_{2}^{2} f^{\prime 4}(\alpha) c_{4}+4 a_{3}^{2} f^{\prime 2}(\alpha) c_{2}^{3}-6 a_{3}^{2} f^{\prime 2}(\alpha) c_{4} \\
&-2 a_{1}^{2} c_{3} c_{2}+2 a_{1}^{2} c_{2}^{3}+32 a_{2}^{2} f^{\prime 4}(\alpha) c_{2}^{3} \\
&-28 c_{2} a_{2} f^{\prime 3}(\alpha) c_{3} a_{3}+34 f^{\prime 3}(\alpha) a_{3} c_{2}^{3} a_{2} \\
&-10 a_{3}^{2} f^{\prime 2}(\alpha) c_{2} c_{3}-6 a_{2} f^{\prime 2}(\alpha) c_{4} a_{1} \\
&\left.+4 a_{3} f^{\prime}(\alpha) c_{2}^{3} a_{1}-6 a_{3} f^{\prime}(\alpha) c_{4} a_{1}\right) e_{n}^{2} \\
& /\left.\left(a_{2} f^{\prime 2}(\alpha)+a_{3} f^{\prime}(\alpha)+a_{1}\right)^{2}+O\left(e_{n}^{3}\right)\right] \\
& \\
&
\end{aligned}
$$

Taking into account (36), (38), (49), and (50), we finally obtain

$$
\begin{array}{r}
x_{n+1} \\
=x_{n}-\left(\left(\left[f^{\prime}\left(x_{n}\right)-p f\left(x_{n}\right)\right]-f^{\prime}\left(x_{n}\right)\right.\right. \\
\left.\quad \times \sum_{k \geq 0}^{m}\left(\begin{array}{c}
\frac{1}{2} \\
k
\end{array}\right)\left(-L_{a_{i}, f, p}\left(x_{n}, w_{n}\right)\right)^{k}\right)
\end{array}
$$

$$
\begin{aligned}
&\left.\times\left(L_{a_{i}}\left(x_{n}, w_{n}\right)+p^{2} f\left(x_{n}\right)-2 p f^{\prime}\left(x_{n}\right)\right)^{-1}\right) \\
&=x_{n}-e_{n}+\frac{1}{2}\left(-40 f^{\prime 3}(\alpha) a_{3} c_{2}^{3} a_{2}-30 a_{1} c_{2}^{3} a_{2} f^{\prime 2}(\alpha)\right. \\
&-2 a_{3} f^{\prime}(\alpha) c_{2}^{3} a_{1}-2 a_{3}^{2} f^{\prime 2}(\alpha) c_{2}^{3} \\
&-4 a_{2}^{2} f^{\prime 4}(\alpha) c_{2}^{3}+2 c_{2}^{2} p a_{1}^{2}+6 p f^{\prime}(\alpha) c_{2}^{2} a_{3} a_{1} \\
&+8 p f^{\prime 2}(\alpha) c_{2}^{2} a_{2} a_{1}+4 p f^{\prime 2}(\alpha) c_{2}^{2} a_{3}^{2} \\
&+10 f^{\prime 3}(\alpha) p c_{2}^{2} a_{2} a_{3}+6 p f^{\prime 4}(\alpha) c_{2}^{2} a_{2}^{2} \\
&-3 p^{2} c_{2} a_{3}^{2} f^{\prime 2}(\alpha)-6 p^{2} c_{2} a_{2} f^{\prime 2}(\alpha) a_{1} \\
&-6 p^{2} c_{2} a_{3} f^{\prime}(\alpha) a_{1}-3 p^{2} c_{2} a_{1}^{2} \\
&-3 p^{2} c_{2} a_{2}^{2} f^{\prime 4}(\alpha)-6 p^{2} c_{2} a_{2} f^{\prime 3}(\alpha) a_{3} \\
&+ 2 f^{\prime 2}(\alpha) p^{3} a_{1} a_{2}+2 f^{\prime 3}(\alpha) p^{3} a_{2} a_{3} \\
&+p^{3} a_{1}^{2}+2 f^{\prime}(\alpha) p^{3} a_{1} a_{3}+f^{\prime 4}(\alpha) p^{3} a_{2}^{2} \\
&\left.+f^{\prime 2}(\alpha) p^{3} a_{3}^{2}\right) e_{n}^{3} /\left(c_{2}-p\right) \\
&\left.f^{\prime}(\alpha)+a_{1}\right)^{2}+O\left(e_{n}^{4}\right) .
\end{aligned}
$$

Taking into account the last expression (51) and $e_{n+1}=x_{n+1}-$ $\alpha$, we have

$$
\begin{aligned}
& e_{n+1}=\frac{1}{2}(-40 f^{\prime 3}(\alpha) a_{3} c_{2}^{3} a_{2}-30 a_{1} c_{2}^{3} a_{2} f^{\prime 2}(\alpha) \\
&-2 a_{3} f^{\prime}(\alpha) c_{2}^{3} a_{1}-2 a_{3}^{2} f^{\prime 2}(\alpha) c_{2}^{3} \\
&-4 a_{2}^{2} f^{\prime 4}(\alpha) c_{2}^{3}+2 c_{2}^{2} p a_{1}^{2}+6 p f^{\prime}(\alpha) c_{2}^{2} a_{3} a_{1} \\
&+8 p f^{\prime 2}(\alpha) c_{2}^{2} a_{2} a_{1}+4 p f^{\prime 2}(\alpha) c_{2}^{2} a_{3}^{2} \\
&+10 f^{\prime 3}(\alpha) p c_{2}^{2} a_{2} a_{3}+6 p f^{\prime 4}(\alpha) c_{2}^{2} a_{2}^{2} \\
&-3 p^{2} c_{2} a_{3}^{2} f^{\prime 2}(\alpha)-6 p^{2} c_{2} a_{2} f^{\prime 2}(\alpha) a_{1} \\
&-6 p^{2} c_{2} a_{3} f^{\prime}(\alpha) a_{1}-3 p^{2} c_{2} a_{1}^{2} \\
&-3 p^{2} c_{2} a_{2}^{2} f^{\prime 4}(\alpha)-6 p^{2} c_{2} a_{2} f^{\prime 3}(\alpha) a_{3} \\
&+2 f^{\prime 2}(\alpha) p^{3} a_{1} a_{2}+2 f^{\prime 3}(\alpha) p^{3} a_{2} a_{3} \\
&+p^{3} a_{1}^{2}+2 f^{\prime}(\alpha) p^{3} a_{1} a_{3}+f^{\prime 4}(\alpha) p^{3} a_{2}^{2} \\
&\left.+f^{\prime 2}(\alpha) p^{3} a_{3}^{2}\right) e_{n}^{3} /\left(c_{2}-p\right) \\
& /\left(a_{2} f^{\prime 2}(\alpha)+a_{3} f^{\prime}(\alpha)+a_{1}\right)^{2}+O\left(e_{n}^{4}\right) .
\end{aligned}
$$


This means that the methods defined by (35) are at least of order three for any $a_{i}(i=1,2,3), p \in R$. Furthermore, we consider that if $m \geq 2, a_{2}=a_{3}=p=0$, then the methods defined by (35) are shown to converge the order four.

\section{Some Special Cases}

From (33)-(35), we have

$$
\begin{gathered}
L_{a_{1}, a_{2}, a_{3}}\left(x_{n}, w_{n}\right) \\
=\left(\left[2 a_{1}+2 a_{2} f^{\prime 2}\left(x_{n}\right)+2 a_{3} f^{\prime}\left(x_{n}\right)\right] f^{\prime 2}\left(x_{n}\right) f\left(w_{n}\right)\right) \\
\times\left(a_{1} f^{2}\left(x_{n}\right)+a_{2} f^{\prime 2}\left(x_{n}\right)\left[f\left(x_{n}\right)-f\left(w_{n}\right)\right]^{2}\right. \\
\left.\quad+a_{3} f\left(x_{n}\right) f^{\prime}\left(x_{n}\right)\left[f\left(x_{n}\right)-f\left(w_{n}\right)\right]\right)^{-1},
\end{gathered}
$$

where $a_{1}, a_{2}, a_{3} \in R$. From (33) we can approximate

$$
\begin{aligned}
L_{a_{1}, a_{2}, a_{3}, f, p}\left(x_{n}, w_{n}\right)= & \left(2 L_{a_{1}, a_{2}, a_{3}}\left(x_{n}, w_{n}\right) f\left(x_{n}\right)\right. \\
& \left.+p^{2} f^{2}\left(x_{n}\right)-2 p f^{\prime}\left(x_{n}\right) f\left(x_{n}\right)\right) \\
& \times\left(f^{\prime 2}\left(x_{n}\right)\right)^{-1} .
\end{aligned}
$$

Let

$$
\begin{aligned}
& K_{a_{1}, a_{2}, a_{3}, f, p}\left(x_{n}, w_{n}\right) \\
& =\left(\left[f^{\prime}\left(x_{n}\right)-p f\left(x_{n}\right)\right]\right. \\
& \left.\quad-f^{\prime}\left(x_{n}\right) \sum_{k \geq 0}^{m}\left(\begin{array}{l}
\frac{1}{2} \\
k
\end{array}\right)\left(-L_{a_{1}, a_{2}, a_{3}, f, p}\left(x_{n}, w_{n}\right)\right)^{k}\right) \\
& \quad \times\left(L_{a_{1}, a_{2}, a_{3}}\left(x_{n}, w_{n}\right)+p^{2} f\left(x_{n}\right)-2 p f^{\prime}\left(x_{n}\right)\right)^{-1},
\end{aligned}
$$

where $a_{1}, a_{2}, a_{3}, p \in R, m \geq 0$.

$1^{0}$ : If $a_{1}=a_{2}=a_{3}=p=1$, and $m=3$, from (55), we obtain a third-order method (LM1):

$$
x_{n+1}=x_{n}-K_{1,1,1, f, 1}\left(x_{n}, w_{n}\right) .
$$

$2^{0}$ : If $a_{1}=a_{2}=0, a_{3}=p=1$, and $m=3$, from (55) we also obtain a third-order method (LM2):

$$
x_{n+1}=x_{n}-K_{0,0,1, f, 1}\left(x_{n}, w_{n}\right) \text {. }
$$

$3^{0}:$ If $a_{1}=1, a_{2}=0, a_{3}=p=1$, and $m=3$, from (55) we obtain a new third-order method (LM3):

$$
x_{n+1}=x_{n}-K_{1,0,1, f, 1}\left(x_{n}, w_{n}\right) .
$$

$4^{0}:$ If $a_{1}=-1, a_{2}=a_{3}=0, p=1$, and $m=3$, from (55) we obtain a third-order method (LM4):

$$
x_{n+1}=x_{n}-K_{-1,0,0, f, 1}\left(x_{n}, w_{n}\right) .
$$

$5^{0}:$ If $a_{1}=a_{2}=0, a_{3}=-1, p=1$, and $m=3$, from (55) we obtain a new third-order method (LM5):

$$
x_{n+1}=x_{n}-K_{0,0,-1, f, 1}\left(x_{n}, w_{n}\right) .
$$

\section{Numerical Examples}

In this section, some numerical examples commonly used in the literature are presented in Table 1 to check the effectiveness of the new methods. The following methods were compared: Newton method (NM), the method of Weerakoon and Fernando [10] (WF), the method of Potra and Pták (PP) [11], Chebyshev's method (CHM) [12, 13], Halley's method (HM) [12], and our new methods (56) (LM1), (57) (LM2), (58) (LM3), (59) (LM4), and (60) (LM5). Displayed in Table 1 are the number of iterations (IT), the number of function evaluations (NFE) counted as the sum of the number of evaluations of the function itself plus the number of evaluations of the derivative, the value $f\left(x_{n+1}\right)$, the computing time (TIME, the unit of time is one second), and the distance of two consecutive approximations $\delta=\left|x_{n+1}-x_{n}\right|$. All computations were done using Matlab 7.1 environment with a ADM athlon (tm) II X2 250-3.01 GHz based PC. We accept an approximate solution rather than the exact root, depending on the precision $\epsilon$ of the computer. We use the following stopping criteria for computer programs: $\left|f\left(x_{n+1}\right)\right|<\epsilon$, we used the fixed stopping criterion $\epsilon=10^{-15}$.

We used the following test functions and display the computed approximate zero $x^{*}[16]$ :

$$
\begin{aligned}
& f_{1}(x)=x^{3}+4 x^{2}-10, \quad x^{*}=1.3652300134140969 \\
& f_{2}(x)=x^{2}-e^{x}-3 x+2, \quad x^{*}=0.25753028543986076 \\
& f_{3}(x)=\sin (x) e^{x}+\ln \left(1+x^{2}\right), \quad x^{*}=0 \\
& f_{4}(x)=(x-1)^{3}-1, \quad x^{*}=2 \\
& f_{5}(x)=\cos x-x, \quad x^{*}=0.73908513321516067 \\
& f_{6}(x)=\sin ^{2} x-x^{2}+1, \quad x^{*}=1.4044916482153411 \\
& f_{7}(x)=e^{x^{2}+7 x-30}-1, \quad x^{*}=3
\end{aligned}
$$

\section{Conclusions}

In this paper, we presented two new families of iterative methods for solving nonlinear equations. One is developed by fitting the model $m(x)=e^{p x}\left(A x^{2}+B x+C\right)$ to the function $f(x)$ and its derivative $f^{\prime}(x), f^{\prime \prime}(x)$ at a point $x_{n}$. The other family of iterative methods was constructed by approximating the equation $f(x)=0$ around the point $\left(x_{n}, f\left(x_{n}\right)\right)$ with the quadratic equation to avoid the calculation of the second derivatives. Analysis of convergence shows that the new methods have third-order or higher convergence: if $m \geq 2, a_{2}=a_{3}=p=0$, then the methods defined by (35) are shown to converge the order four. We observed from numerical examples that the proposed methods are 
TABLE 1: Comparison of various third-order methods and Newton's method.

\begin{tabular}{|c|c|c|c|c|c|}
\hline & IT & NFE & $f\left(x_{n+1}\right)$ & Time & $\delta$ \\
\hline \multicolumn{6}{|c|}{$f_{1}: x_{0}=1$} \\
\hline NM & 5 & 10 & 0 & 0.062577 & $2.126987475037367 e-011$ \\
\hline WF & 3 & 9 & 0 & 0.018731 & $2.284722713019605 e-006$ \\
\hline $\mathrm{PP}$ & 4 & 12 & 0 & 0.044520 & $1.558753126573720 e-013$ \\
\hline $\mathrm{CHM}$ & 4 & 12 & 0 & 0.040102 & $1.643130076445232 e-014$ \\
\hline $\mathrm{HM}$ & 3 & 9 & 0 & 0.019876 & $3.698649917449615 e-007$ \\
\hline LM1 & 4 & 12 & 0 & 0.029137 & $1.043609643147647 e-014$ \\
\hline LM2 & 3 & 9 & 0 & 0.022242 & $1.788683664960544 e-006$ \\
\hline LM3 & 3 & 9 & 0 & 0.023550 & $1.295199573814188 e-006$ \\
\hline LM4 & 3 & 9 & 0 & 0.019227 & $3.091731315407742 e-010$ \\
\hline LM5 & 3 & 9 & 0 & 0.020131 & $1.788683664960544 e-006$ \\
\hline \multicolumn{6}{|c|}{$f_{1}: x_{0}=2$} \\
\hline NM & 5 & 10 & 0 & 0.057231 & $5.020497351182485 e-010$ \\
\hline WF & 4 & 12 & 0 & 0.039384 & $4.440892098500626 e-016$ \\
\hline $\mathrm{PP}$ & 4 & 12 & 0 & 0.044239 & $7.949196856316121 e-014$ \\
\hline $\mathrm{CHM}$ & 4 & 12 & 0 & 0.042782 & $2.065014825802791 e-014$ \\
\hline $\mathrm{HM}$ & 3 & 9 & 0 & 0.017733 & $3.107350415199051 e-006$ \\
\hline LM1 & 4 & 12 & 0 & 0.032638 & $2.706235235905297 e-011$ \\
\hline LM2 & 4 & 12 & 0 & 0.037830 & $5.551115123125783 e-015$ \\
\hline LM3 & 4 & 12 & 0 & 0.034087 & $3.108624468950438 e-015$ \\
\hline LM4 & 4 & 12 & 0 & 0.038399 & $2.220446049250313 e-016$ \\
\hline LM5 & 4 & 12 & 0 & 0.035809 & $5.551115123125783 e-015$ \\
\hline \multicolumn{6}{|c|}{$f_{2}: x_{0}=0$} \\
\hline NM & 4 & 8 & 0 & 0.035702 & $2.665312415217613 e-012$ \\
\hline WF & 3 & 9 & 0 & 0.019699 & $7.801814749797131 e-012$ \\
\hline $\mathrm{PP}$ & 3 & 9 & 0 & 0.021596 & $1.219191414492116 e-012$ \\
\hline $\mathrm{CHM}$ & 3 & 9 & 0 & 0.023312 & $8.906764215055318 e-013$ \\
\hline $\mathrm{HM}$ & 3 & 9 & 0 & 0.017431 & $7.374600929921371 e-012$ \\
\hline LM1 & 3 & 9 & 0 & 0.016351 & $9.004856806882344 e-007$ \\
\hline LM2 & 3 & 9 & 0 & 0.017242 & $8.067152316715287 e-007$ \\
\hline LM3 & 3 & 9 & 0 & 0.018978 & $8.334935165388302 e-007$ \\
\hline LM4 & 3 & 9 & 0 & 0.016178 & $7.334822825222354 e-007$ \\
\hline LM5 & 3 & 9 & 0 & 0.016795 & $8.067152316715287 e-007$ \\
\hline \multicolumn{6}{|c|}{$f_{2}: x_{0}=0.5$} \\
\hline NM & 4 & 8 & 0 & 0.044392 & $1.791899961745003 e-013$ \\
\hline WF & 3 & 9 & 0 & 0.023132 & $6.424749621203318 e-012$ \\
\hline $\mathrm{PP}$ & 3 & 9 & 0 & 0.024293 & $4.607425552194400 e-014$ \\
\hline $\mathrm{CHM}$ & 3 & 9 & 0 & 0.026689 & $3.087480271446452 e-011$ \\
\hline $\mathrm{HM}$ & 3 & 9 & 0 & 0.022199 & $4.208039472430869 e-011$ \\
\hline LM1 & 3 & 9 & 0 & 0.024120 & $2.850281847210923 e-007$ \\
\hline LM2 & 3 & 9 & $4.440892098500626 e-016$ & 0.021035 & $2.744941123289379 e-007$ \\
\hline LM3 & 3 & 9 & 0 & 0.021310 & $2.778229745148408 e-007$ \\
\hline LM4 & 3 & 9 & 0 & 0.020886 & $2.655207446689012 e-007$ \\
\hline LM5 & 3 & 9 & $4.440892098500626 e-016$ & 0.021592 & $2.744941123289379 e-007$ \\
\hline \multicolumn{6}{|c|}{$f_{3}: x_{0}=1$} \\
\hline NM & 7 & 14 & $3.537126081266182 e-024$ & 0.085178 & $1.085848323840232 e-012$ \\
\hline WF & 4 & 12 & $2.621304391538411 e-016$ & 0.030341 & $4.330310691887267 e-006$ \\
\hline $\mathrm{PP}$ & 5 & 15 & $8.196910187379942 e-033$ & 0.063971 & $8.806888499109001 e-012$ \\
\hline $\mathrm{CHM}$ & 5 & 15 & $6.352230116524407 e-022$ & 0.053064 & $2.520356663650445 e-011$ \\
\hline $\mathrm{HM}$ & 5 & 15 & $7.257520328033309 e-029$ & 0.055315 & $8.459855063117184 e-015$ \\
\hline LM1 & 5 & 15 & $5.355132874954819 e-017$ & 0.041370 & $4.825251676864564 e-007$ \\
\hline LM2 & 4 & 12 & $6.348042447121620 e-018$ & 0.026722 & $5.276208195236892 e-013$ \\
\hline LM3 & 3 & 9 & $1.559423551656343 e-017$ & 0.019476 & $5.545966966175517 e-011$ \\
\hline LM4 & 4 & 12 & $6.485252546346663 e-018$ & 0.030513 & $1.317913979899399 e-009$ \\
\hline LM5 & 4 & 12 & $6.348042447121620 e-018$ & 0.031575 & $5.276208195236892 e-013$ \\
\hline
\end{tabular}


TABLe 1: Continued.

\begin{tabular}{|c|c|c|c|c|c|}
\hline & IT & NFE & $f\left(x_{n+1}\right)$ & Time & $\delta$ \\
\hline \multicolumn{6}{|c|}{$f_{3}: x_{0}=0.5$} \\
\hline NM & 6 & 12 & $5.905159674954809 e-020$ & 0.071738 & $1.402992074360412 e-010$ \\
\hline WF & 4 & 12 & $1.764824578467612 e-017$ & 0.039948 & $4.200981459101664 e-009$ \\
\hline $\mathrm{PP}$ & 4 & 12 & $1.694834561079519 e-019$ & 0.056046 & $2.767209186089879 e-007$ \\
\hline $\mathrm{CHM}$ & 4 & 12 & $8.798671206634291 e-017$ & 0.036458 & $3.059650585008672 e-007$ \\
\hline $\mathrm{HM}$ & 4 & 12 & $3.044907255908736 e-017$ & 0.072238 & $5.518067735074518 e-009$ \\
\hline LM1 & 4 & 12 & $1.293991275513200 e-017$ & 0.033751 & $1.322689611777333 e-013$ \\
\hline LM2 & 4 & 12 & $2.828488650436813 e-016$ & 0.032997 & $4.626219030783813 e-006$ \\
\hline LM3 & 4 & 12 & $6.789745028898642 e-018$ & 0.036425 & $1.912594303370619 e-014$ \\
\hline LM4 & 4 & 12 & $1.506225012219799 e-017$ & 0.034137 & $2.365541379047147 e-011$ \\
\hline LM5 & 4 & 12 & $2.828488650436813 e-016$ & 0.035087 & $4.626219030783813 e-006$ \\
\hline \multicolumn{6}{|c|}{$f_{4}: x_{0}=2.5$} \\
\hline NM & 6 & 12 & 0 & 0.071353 & $1.154631945610163 e-014$ \\
\hline WF & 4 & 12 & 0 & 0.034140 & $7.314593375440381 e-012$ \\
\hline $\mathrm{PP}$ & 4 & 12 & 0 & 0.042598 & $4.221685223626537 e-010$ \\
\hline $\mathrm{CHM}$ & 4 & 12 & 0 & 0.036365 & $9.853584614916144 e-011$ \\
\hline $\mathrm{HM}$ & 4 & 12 & 0 & 0.038080 & $4.662936703425658 e-014$ \\
\hline LM1 & 3 & 9 & 0 & 0.018372 & $9.336336148635382 e-010$ \\
\hline LM2 & 4 & 12 & 0 & 0.035562 & $1.610311883837312 e-010$ \\
\hline LM3 & 4 & 12 & 0 & 0.036985 & $2.312174895990893 e-010$ \\
\hline LM4 & 4 & 12 & 0 & 0.035463 & $5.127454016928823 e-012$ \\
\hline LM5 & 4 & 12 & 0 & 0.033362 & $1.610311883837312 e-010$ \\
\hline \multicolumn{6}{|c|}{$f_{4}: x_{0}=3.5$} \\
\hline NM & 7 & 14 & 0 & 2.151288 & $2.877564853065451 e-011$ \\
\hline WF & 5 & 15 & 0 & 0.054803 & $6.550315845288424 e-013$ \\
\hline $\mathrm{PP}$ & 5 & 15 & 0 & 0.063380 & $4.512221707386743 e-010$ \\
\hline $\mathrm{CHM}$ & 5 & 15 & 0 & 0.061753 & $4.188738245147761 e-011$ \\
\hline $\mathrm{HM}$ & 4 & 12 & 0 & 0.046608 & $4.485352507632712 e-006$ \\
\hline LM1 & 5 & 15 & $6.661338147750939 e-016$ & 0.050909 & $2.781577124189028 e-008$ \\
\hline LM2 & 5 & 15 & 0 & 0.048688 & $9.426681657487279 e-011$ \\
\hline LM3 & 10 & 30 & 0 & 0.111640 & $1.081885248055414 e-009$ \\
\hline LM4 & 4 & 12 & 0 & 0.040109 & $2.154232348061669 e-011$ \\
\hline LM5 & 5 & 15 & 0 & 0.043482 & $9.426681657487279 e-011$ \\
\hline \multicolumn{6}{|c|}{$f_{5}: x_{0}=0$} \\
\hline NM & 5 & 10 & 0 & 0.050123 & $1.701233598438989 e-010$ \\
\hline WF & 3 & 9 & 0 & 0.023397 & $7.792236328407753 e-007$ \\
\hline $\mathrm{PP}$ & 4 & 12 & 0 & 0.038373 & $1.500558566291943 e-010$ \\
\hline $\mathrm{CHM}$ & 4 & 12 & 0 & 0.037498 & $5.327979279989847 e-009$ \\
\hline $\mathrm{HM}$ & 4 & 12 & 0 & 0.035701 & $1.121325254871408 e-014$ \\
\hline LM1 & 4 & 12 & $3.330669073875470 e-016$ & 0.028362 & $4.678117765610779 e-006$ \\
\hline LM2 & 4 & 12 & 0 & 0.028666 & $7.181037887660224 e-007$ \\
\hline LM3 & 6 & 18 & 0 & 0.053924 & $6.676881270095691 e-013$ \\
\hline LM4 & 4 & 12 & 0 & 0.028247 & $2.163133006050089 e-010$ \\
\hline LM5 & 4 & 12 & 0 & 0.029150 & $7.181037887660224 e-007$ \\
\hline \multicolumn{6}{|c|}{$f_{5}: x_{0}=1$} \\
\hline $\mathrm{NM}$ & 4 & 8 & 0 & 0.039328 & $1.701233598438989 e-010$ \\
\hline WF & 2 & 6 & $4.440892098500626 e-016$ & 0.003092 & $2.674277017133964 e-005$ \\
\hline $\mathrm{PP}$ & 3 & 9 & 0 & 0.020461 & $9.809075773858922 e-011$ \\
\hline $\mathrm{CHM}$ & 3 & 9 & 0 & 0.019378 & $1.600380383770528 e-009$ \\
\hline $\mathrm{HM}$ & 3 & 9 & 0 & 0.018186 & $6.624212289807474 e-010$ \\
\hline LM1 & 3 & 9 & 0 & 0.018199 & $7.500783549829748 e-008$ \\
\hline LM2 & 3 & 9 & $1.110223024625157 e-016$ & 0.017732 & $5.330569952111119 e-008$ \\
\hline LM3 & 3 & 9 & 0 & 0.013523 & $8.804162354714151 e-008$ \\
\hline LM4 & 3 & 9 & 0 & 0.013782 & $3.531516445942629 e-008$ \\
\hline LM5 & 3 & 9 & $1.110223024625157 e-016$ & 0.016581 & $5.330569952111119 e-008$ \\
\hline
\end{tabular}


TABle 1: Continued.

\begin{tabular}{|c|c|c|c|c|c|}
\hline & IT & NFE & $f\left(x_{n+1}\right)$ & Time & $\delta$ \\
\hline \multicolumn{6}{|c|}{$f_{6}: x_{0}=1$} \\
\hline NM & 6 & 12 & $3.330669073875470 e-016$ & 0.065520 & $3.059774655866931 e-013$ \\
\hline WF & 4 & 12 & $4.440892098500626 e-016$ & 0.030929 & $1.793023507445923 e-010$ \\
\hline PP & 16 & 48 & $4.440892098500626 e-016$ & 0.266014 & $1.531728257564424 e-007$ \\
\hline $\mathrm{CHM}$ & 5 & 15 & $4.440892098500626 e-016$ & 0.061006 & $6.883094094689568 e-010$ \\
\hline $\mathrm{HM}$ & 4 & 12 & $4.440892098500626 e-016$ & 0.043655 & $2.686739719592879 e-013$ \\
\hline LM1 & 4 & 12 & $3.330669073875470 e-016$ & 0.030877 & $1.659126835917846 e-008$ \\
\hline LM2 & 4 & 12 & $3.330669073875470 e-016$ & 0.031156 & $9.103828801926284 e-014$ \\
\hline LM3 & 4 & 12 & $3.330669073875470 e-016$ & 0.033076 & $1.261090962767497 e-006$ \\
\hline LM4 & 5 & 15 & $4.440892098500626 e-016$ & 0.041102 & $8.215650382226158 e-014$ \\
\hline LM5 & 4 & 12 & $3.330669073875470 e-016$ & 0.030923 & $9.103828801926284 e-014$ \\
\hline \multicolumn{6}{|c|}{$f_{6}: x_{0}=2.5$} \\
\hline NM & 6 & 12 & $3.330669073875470 e-016$ & 0.064979 & $1.404654170755748 e-012$ \\
\hline WF & 4 & 12 & $3.330669073875470 e-016$ & 0.038404 & $4.229505634611996 e-012$ \\
\hline PP & 4 & 12 & $3.330669073875470 e-016$ & 0.042164 & $1.030850205196998 e-008$ \\
\hline $\mathrm{CHM}$ & 4 & 12 & $3.330669073875470 e-016$ & 0.043437 & $1.475204565171140 e-007$ \\
\hline $\mathrm{HM}$ & 4 & 12 & $4.440892098500626 e-016$ & 0.043896 & $9.462626682221753 e-009$ \\
\hline LM1 & 5 & 15 & $3.330669073875470 e-016$ & 0.053132 & $6.201483770951199 e-012$ \\
\hline LM2 & 4 & 12 & $3.330669073875470 e-016$ & 0.036191 & $4.450250368215336 e-008$ \\
\hline LM3 & 6 & 18 & $3.330669073875470 e-016$ & 0.064485 & $8.881784197001252 e-016$ \\
\hline LM4 & 4 & 12 & $3.330669073875470 e-016$ & 0.035393 & $5.568381311604753 e-009$ \\
\hline LM5 & 4 & 12 & $3.330669073875470 e-016$ & 0.036946 & $4.450250368215336 e-008$ \\
\hline \multicolumn{6}{|c|}{$f_{7}: x_{0}=3.25$} \\
\hline NM & 8 & 16 & 0 & 0.114189 & $9.720393379097914 e-010$ \\
\hline WF & 6 & 18 & 0 & 0.065645 & $1.691979889528739 e-013$ \\
\hline $\mathrm{PP}$ & 6 & 18 & 0 & 0.073469 & $1.131490456884876 e-010$ \\
\hline $\mathrm{CHM}$ & 6 & 18 & 0 & 0.082677 & $2.398081733190338 e-014$ \\
\hline HM & 5 & 15 & 0 & 0.055630 & $3.082423205569285 e-012$ \\
\hline LM1 & 4 & 12 & 0 & 0.036191 & $2.613820271335499 e-011$ \\
\hline LM2 & 5 & 15 & 0 & 0.049599 & $4.440892098500626 e-016$ \\
\hline LM3 & 4 & 12 & 0 & 0.038172 & $1.546353226355990 e-006$ \\
\hline LM4 & 6 & 18 & 0 & 0.051577 & $4.440892098500626 e-016$ \\
\hline LM5 & 5 & 15 & 0 & 0.048340 & $4.440892098500626 e-016$ \\
\hline \multicolumn{6}{|c|}{$f_{7}: x_{0}=3.45$} \\
\hline NM & 11 & 22 & 0 & 0.147308 & $4.008793297316515 e-011$ \\
\hline WF & 8 & 24 & 0 & 0.106276 & $7.105427357601002 e-015$ \\
\hline $\mathrm{PP}$ & 8 & 24 & 0 & 0.116999 & $2.160227552394645 e-010$ \\
\hline $\mathrm{CHM}$ & 7 & 21 & 0 & 0.100725 & $1.268533917908599 e-006$ \\
\hline $\mathrm{HM}$ & 6 & 18 & 0 & 0.062876 & $1.694565332499565 e-008$ \\
\hline LM1 & 6 & 18 & 0 & 0.060920 & $4.440892098500626 e-016$ \\
\hline LM2 & 6 & 18 & 0 & 0.060951 & $1.753264200488047 e-011$ \\
\hline LM3 & 6 & 18 & 0 & 0.060627 & $1.518252190635394 e-011$ \\
\hline LM4 & 7 & 21 & 0 & 0.087873 & $9.144596191390519 e-011$ \\
\hline LM5 & 6 & 18 & 0 & 0.060607 & $1.753264200488047 e-011$ \\
\hline
\end{tabular}

efficient and demonstrate equal or better performance as compared with other well-known methods.

\section{References}

[1] A. M. Ostrowski, Solution of Equations in Euclidean and Banach Spaces, Academic Press, New York, NY, USA, 1973.

[2] J. F. Traub, Iterative Methods for the Solution of Equations, AMS Chelsea, New York, NY, USA, 1977.
[3] J. R. Sharma, "A family of Newton-like methods based on an exponential model," International Journal of Computer Mathematics, vol. 84, no. 3, pp. 297-304, 2007.

[4] C. Chun, "Some second-derivative-free variants of ChebyshevHalley methods," Applied Mathematics and Computation, vol. 191, no. 2, pp. 410-414, 2007.

[5] J. Kou, "Fourth-order variants of Cauchy's method for solving non-linear equations," Applied Mathematics and Computation, vol. 192, no. 1, pp. 113-119, 2007. 
[6] C. Chun, "Some variants of Chebyshev-Halley methods free from second derivative," Applied Mathematics and Computation, vol. 191, no. 1, pp. 193-198, 2007.

[7] S. K. Khattri and I. K. Argyros, "Sixth order derivative free family of iterative methods," Applied Mathematics and Computation, vol. 217, no. 12, pp. 5500-5507, 2011.

[8] T. Liu and H. Li, "Some new variants of Cauchy's methods for solving non-linear equations," Journal of Applied Mathematics, vol. 2012, Article ID 927450, 13 pages, 2012.

[9] S. K. Khattri and I. K. Argyros, "How to develop fourth and seventh order iterative methods?" Novi Sad Journal of Mathematics, vol. 40, no. 2, pp. 61-67, 2010.

[10] S. Weerakoon and T. G. I. Fernando, "A variant of Newton's method with accelerated third-order convergence," Applied Mathematics Letters, vol. 13, no. 8, pp. 87-93, 2000.

[11] F. A. Potra and V. Pták, Nondiscrete Induction and Iterative Processes, vol. 103 of Research Notes in Mathematics, Pitman, Boston, Mass, USA, 1984.

[12] J. M. Gutiérrez and M. A. Hernández, "A family of ChebyshevHalley type methods in Banach spaces," Bulletin of the Australian Mathematical Society, vol. 55, no. 1, pp. 113-130, 1997.

[13] J. Kou, Y. Li, and X. Wang, "On a family of second-derivativefree variants of Chebyshev's method," Applied Mathematics and Computation, vol. 181, no. 2, pp. 982-987, 2006.

[14] Z. Xiaojian, "Modified Chebyshev-Halley methods free from second derivative," Applied Mathematics and Computation, vol. 203, no. 2, pp. 824-827, 2008.

[15] J. Kou and Y. Li, "Modified Chebyshev's method free from second derivative for non-linear equations," Applied Mathematics and Computation, vol. 187, no. 2, pp. 1027-1032, 2007.

[16] S. K. Khattri, M. A. Noor, and E. Al-Said, "Unifying fourthorder family of iterative methods," Applied Mathematics Letters, vol. 24, no. 8, pp. 1295-1300, 2011.

[17] M. A. Noor, "Some iterative methods free from second derivatives for nonlinear equations," Applied Mathematics and Computation, vol. 192, no. 1, pp. 101-106, 2007.

[18] H. T. Kung and J. F. Traub, "Optimal order of one-point and multipoint iteration," Journal of the Association for Computing Machinery, vol. 21, pp. 643-651, 1974.

[19] I. K. Argyros, D. Chen, and Q. Qian, "The Jarratt method in Banach space setting," Journal of Computational and Applied Mathematics, vol. 51, no. 1, pp. 103-106, 1994.

[20] A. Cordero, J. L. Hueso, E. Martínez, and J. R. Torregrosa, "Efficient high-order methods based on golden ratio for nonlinear systems," Applied Mathematics and Computation, vol. 217, no. 9, pp. 4548-4556, 2011.

[21] B. Neta, C. Chun, and M. Scott, "A note on the modified superHalley method," Applied Mathematics and Computation, vol. 218, no. 18, pp. 9575-9577, 2012. 


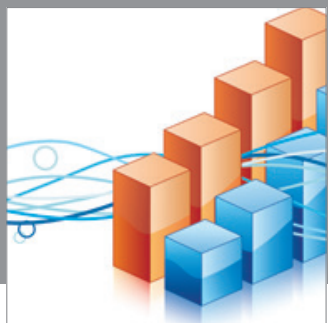

Advances in

Operations Research

mansans

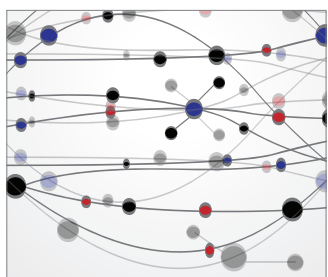

The Scientific World Journal
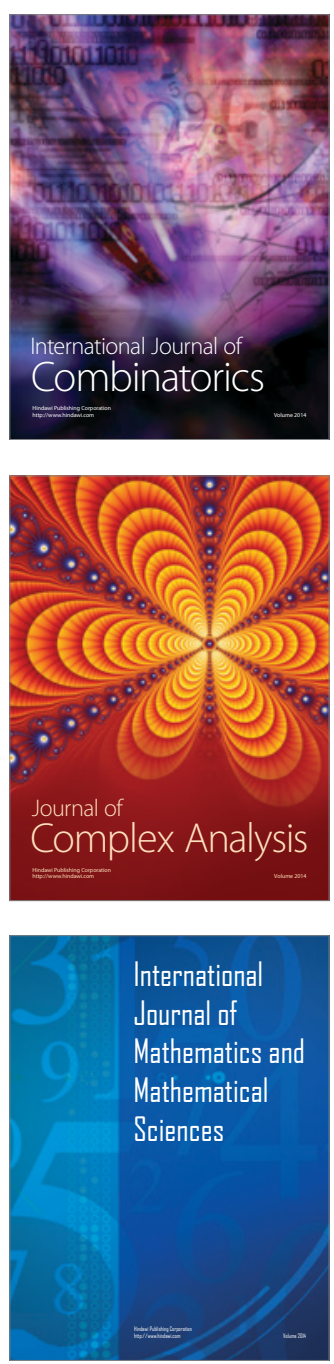
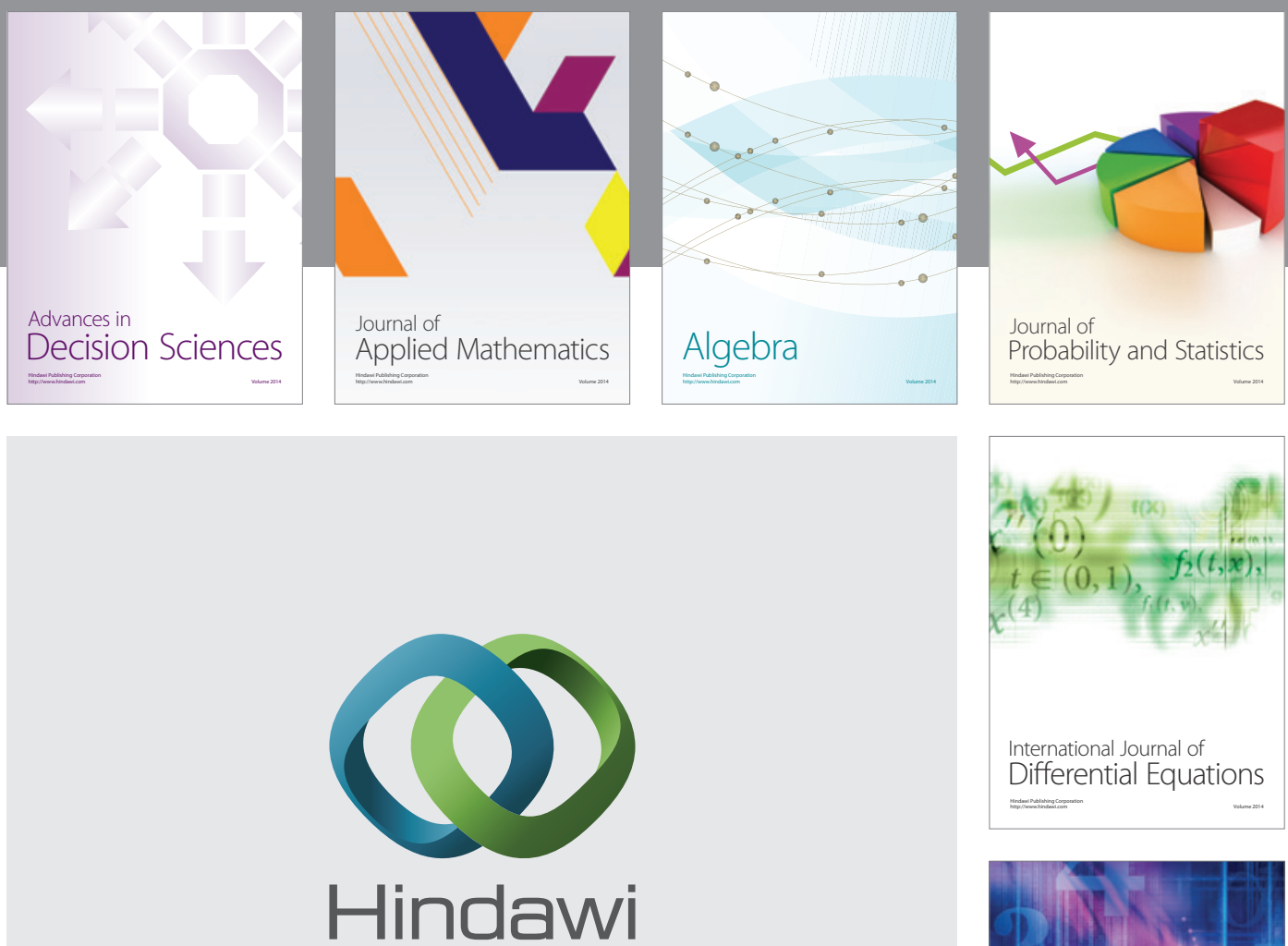

Submit your manuscripts at http://www.hindawi.com
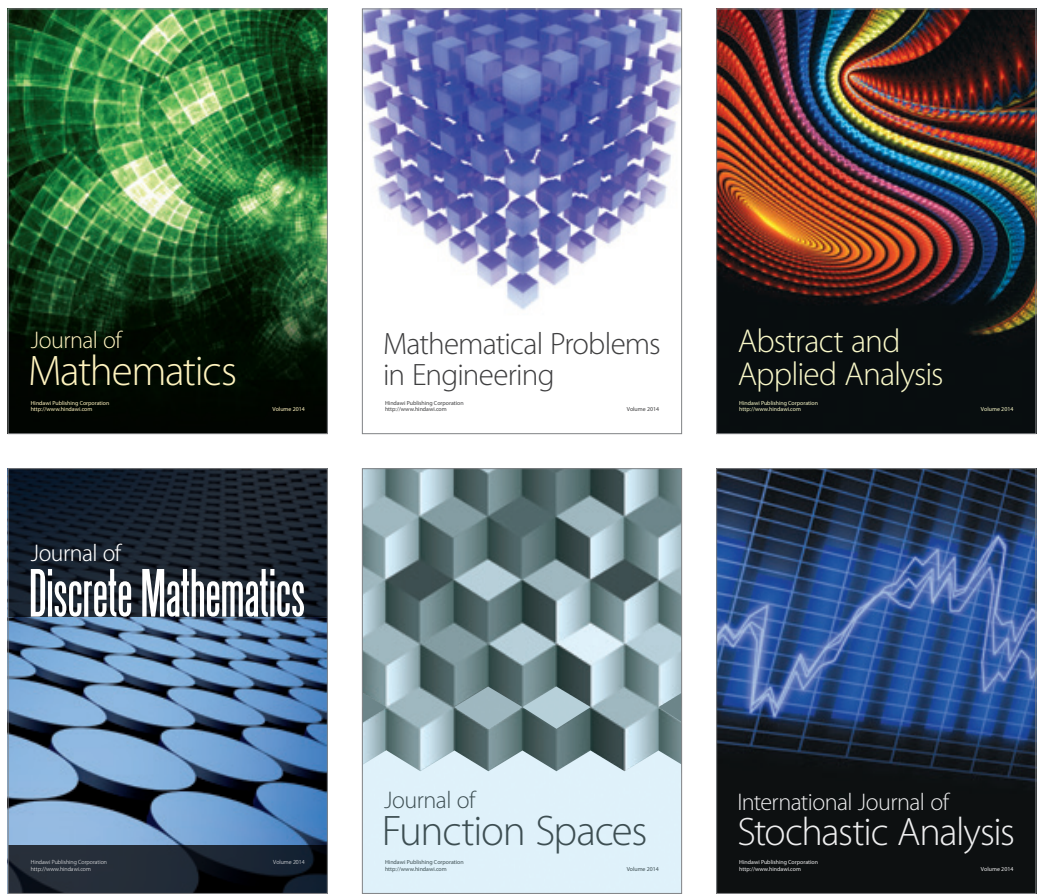

Journal of

Function Spaces

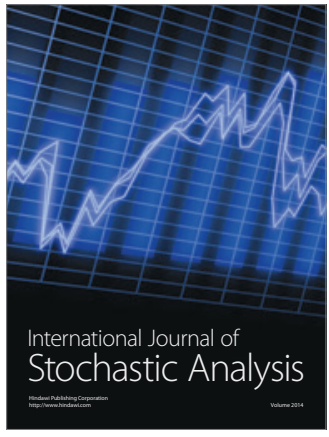

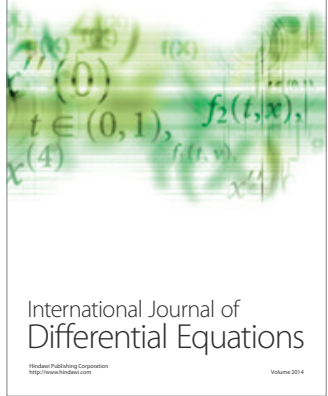
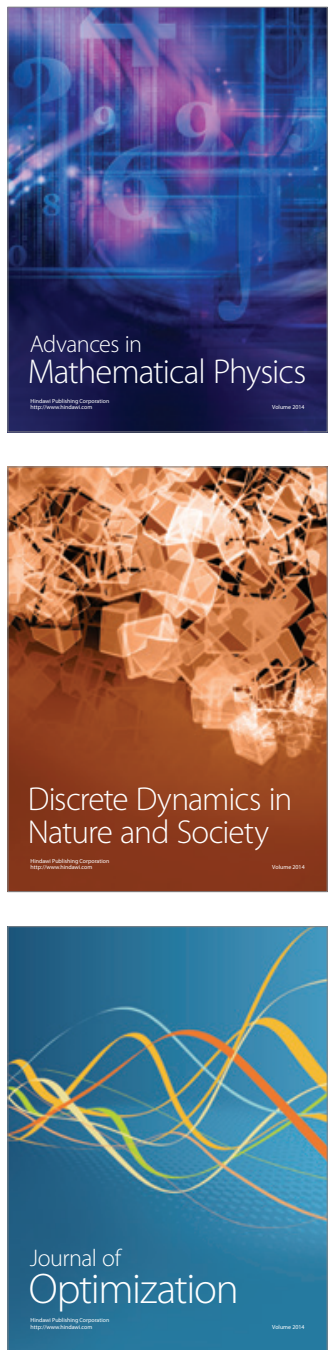